\title{
Effect of intraoperative neuromonitoring on efficacy and safety using sugammadex in thyroid surgery: randomized clinical trial
}

\author{
Mehmet Emin Gunes ${ }^{1}$, Ahmet Cem Dural', Cevher Akarsu', Deniz Guzey ${ }^{1}$, Nuri Alper Sahbaz', \\ Evrim Kucur Tulubas ${ }^{2}$, Sezer Bulut ${ }^{1}$, Turgut Donmez ${ }^{1}$ \\ ${ }^{1}$ Department of General Surgery, Bakırköy Dr.Sadi Konuk Training and Research Hospital, Istanbul, Turkey \\ ${ }^{2}$ Department of Anesthesiology, Bakırköy Dr. Sadi Konuk Training and Research Hospital, Istanbul, Turkey
}

\begin{abstract}
Purpose: The use of nondepolarizing neuromuscular blocking agents (NMBAs) may affect intraoperative neuromonitoring (IONM) during anesthesia used during thyroid and parathyroid surgery.

Methods: The use of sugammadex was evaluated in a prospective clinical study during thyroid surgery. Between July 2018 and January 2019, 129 patients were prospectively randomized to either the sugammadex group (group B) or the control group (group A). Group A patients underwent standardized IONM during thyroidectomy, while group B patients used an NMBA-reversal protocol comprised of rocuronium $(0.6 \mathrm{mg} / \mathrm{kg})$ in anesthesia induction and sugammadex $(2 \mathrm{mg} / \mathrm{kg})$ after first vagal stimulation (VO). A peripheral nerve stimulator was used to monitor the neuromuscular transmission.

Results: In our clinical study, it took $26.07 \pm 3.26$ and $50.0 \pm 8.46$ minutes to reach $100 \%$ recovery of laryngeal electromyography at injection of the sugammadex group $(2 \mathrm{mg} / \mathrm{kg})$ and the control group, respectively $(\mathrm{P}<0.001)$. The train-of-four ratio recovered from 0 to $>0.9$ within 4 minutes after administering $2 \mathrm{mg} / \mathrm{kg}$ of sugammadex at the beginning of resection. Surgery time was significantly shorter in group $B$ than in group $A(P<0.001)$. Transient recurrent laryngeal nerve (RLN) paralysis was detected in 4 patients from group $A$ and in 3 patients from group $B(P=0.681)$. There was no permanent RLN paralysis in the 2 groups.

Conclusion: Our clinical study showed that sugammadex effectively and rapidly improved the inhibition of neuromuscular function induced by rocuronium. The implementation of the nondepolarizing neuromuscular block recovery protocol may lead to tracheal intubation as well as favorable conditions for IONM in thyroid surgery.

[Ann Surg Treat Res 2019;97(6):282-290]
\end{abstract}

Key Words: Neuromuscular blockade, Recurrent laryngeal nerve, Sugammadex, Thyroidectomy

\section{INTRODUCTION}

Thyroid gland surgery has possible serious complications because of the endocrine function of the thyroid gland and its close proximity with critical structures. Despite recent advances in surgical techniques, recurrent laryngeal nerve (RLN) damage has not decreased during thyroid surgery [1]. The incidence of RLN paralysis depends on the type of thyroid disease, surgical technique, previous neck surgery, and the experience of the surgeon $(1 \%-20 \%)[2,3]$. RLN dysfunction is a serious outcome with voice impairment, which leads to disabled vocal communication and causes various social, psychological and occupational problems [3].

The definition of intraoperative RLN was suggested to be an
Received August 28, 2019, Revised October 9, 2019,

Accepted October 23, 2019

\section{Corresponding Author:Turgut Donmez}

Department of General Surgery, Bakirkoy Dr.Sadi Konuk Training and Research Hospital, 223.cd. 23G B2 D36 Halkalı, 34300 Istanbul, Turkey

Tel: +90-212-414-7171, Fax: +90-212-414-6494

E-mail: surgeont73@hotmail.com

ORCID: https://orcid.org/0000-0003-3095-2195

\section{Copyright (c) 2019, the Korean Surgical Society}

(c) Annals of Surgical Treatment and Research is an Open Access Journal. All articles are distributed under the terms of the Creative Commons Attribution NonCommercial License (http://creativecommons.org/licenses/by-nc/4.0/) which permits unrestricted non-commercial use, distribution, and reproduction in any medium, provided the original work is properly cited. 
important part of thyroid surgery in 1938 and was shown to reduce the incidence of postoperative nerve palsy [3-5]. Plenty of studies correlated with this proposal, and routine nerve identification has been suggested as the gold standard for safe thyroid surgery [3-5]. However, even very experienced surgeons might injure the RLN. Permanent and temporary RLN paralysis incidences following thyroid surgery are high (i.e., 2.3\% and 9.8\%, respectively) [6]. In spite of the low rate of permanent damage, it is still an important concern for both patients and surgeons due to malpractice litigation [7].

Nerve visualization is typically employed to protect the RLN [8]. However, the visualized RLN may not be a functional nerve. A structurally intact RLN may not always indicate a functional nerve, which may mislead the surgeon. Postoperative identification of the RLN has been shown to reduce the risk of temporary and permanent nerve palsy. Various neural tracing methods such as direct visualization of the vocal cords (VCs) and nerve monitoring during thyroid surgery have been described [9]. Intraoperative neuromonitoring (IONM) is increasingly used by surgeons conducting thyroid surgeries worldwide. Recent research shows that $53 \%$ of general surgeons in the United States and $65 \%$ of otolaryngologists currently use IONM [8]. IONM has been suggested as an assistant tool to detect the RLN and the external branch of superior laryngeal nerve in thyroid surgery as it provides a decreased injury risk $[5,6,10]$. However, its benefit in reducing RLN damage and its value for predicting postoperative RLN function remains controversial [3,5]. A high negative predictive value and low and highly variable positive predictive values for IONM have been reported in recent studies-92\%-100\% and 10\%-90\%, respectively [10].

Neuromuscular blocking agents (NMBAs) are used during tracheal intubation to ease the process. Of them, rocuronium and vecuronium are preferred over succinylcholine, which is a depolarizing agent with many side effects [11]. In addition to making endotracheal intubation easier and reducing dependent laryngeal morbidity, NMBAs may also decrease electromyography (EMG) signals, interfering with IONM. Because of this, the use of NMBAs for easier intubation in thyroid surgery has been contested [3,12-14]. The peripheral nerve stimulator (PNS) is used for the reversal of NMB, and a tactile or a visual solution to the PNS is observed. By using the PNS monitor to assess the time for a pharmacological recovery, the incidence and severity of permanent RLN paralysis can be decreased. Studies reported that the train-of-four (TOF) ratio should be at least 0.90 so that a complete reversal of the NMB effect on the respiratory muscles can occur, and a normal hypoxic respiratory answer can be maintained $[15,16]$.

Sugammadex is a selective relaxation agent that binds with NMBA (rocuronium and vecuronium); thus, it inhibits the binding of NMBAs to receptors at the neuromuscular junction
[17]. Its efficacy on reversing rocuronium block was evaluated by a recovery of the TOF response, and it was shown that sugammadex effectively reversed intense NMB within 2 to 3 minutes of its administration $[15,16,18]$.

The aim of this clinical study was to evaluate the efficacy and safety of sugammadexin used to block the effect of NMBA during thyroid nerve monitoring surgery.

\section{METHODS}

\section{Study design}

This study was approved by the Institutional Review Board of the Bakırköy Dr. Sadi Konuk Training and Research Hospital (approval number: 2018-261), and written informed consent was obtained from all subjects participating in the trial. The trial was registered prior to patient enrollment at clinicaltrials.gov (NCT03634956, Principal investigator: Turgut Donmez, Date of registration: 08/13/2018). One hundred twenty-nine patients were found suitable for the clinical trial (Fig. 1). A detailed explanation of the procedure and risks involved were given. This prospective, randomized clinical trial was conducted at a training and research hospital in a period of 7 months from 15 August 2018 to 31 January 2019.

\section{Patient selection}

Consecutive, newly diagnosed cases of thyroid surgery that were reported to the surgery department and met the following inclusion criteria were included in the study: American Society of Anesthesiologist (ASA) physical status classification I, II, or III; aged between 18 and 80 years old; multinodular goiter; substernal goiter; differentiated thyroid carcinoma (DTC); toxic goiter; and recurrent multinodular goiter.

Exclusion criteria included patients with RLN paralysis, pregnancy, $<18$ years of age or $>80$ years of age, ASA physical status classification IV.

\section{Methodology}

All of the patients met with the anesthesiologist and the endocrine surgeon and received special information about possible surgical and anesthetic risks before their surgery. Information regarding if and when intravenous medications would be administered were also provided.

The VC examination was performed by an otolaryngolist on preoperative day 1 as well as postoperative day 1 using a flexible laryngoscope. Patients who were diagnosed with RLN paralysis in the postoperative period underwent an additional VC examination at 1, 2, 4, and 6 months. RLN paralysis that continued more than 6 months was accepted as permanent paralysis.

Randomization was performed by computer with a randomization table, which was formed after the patients were 


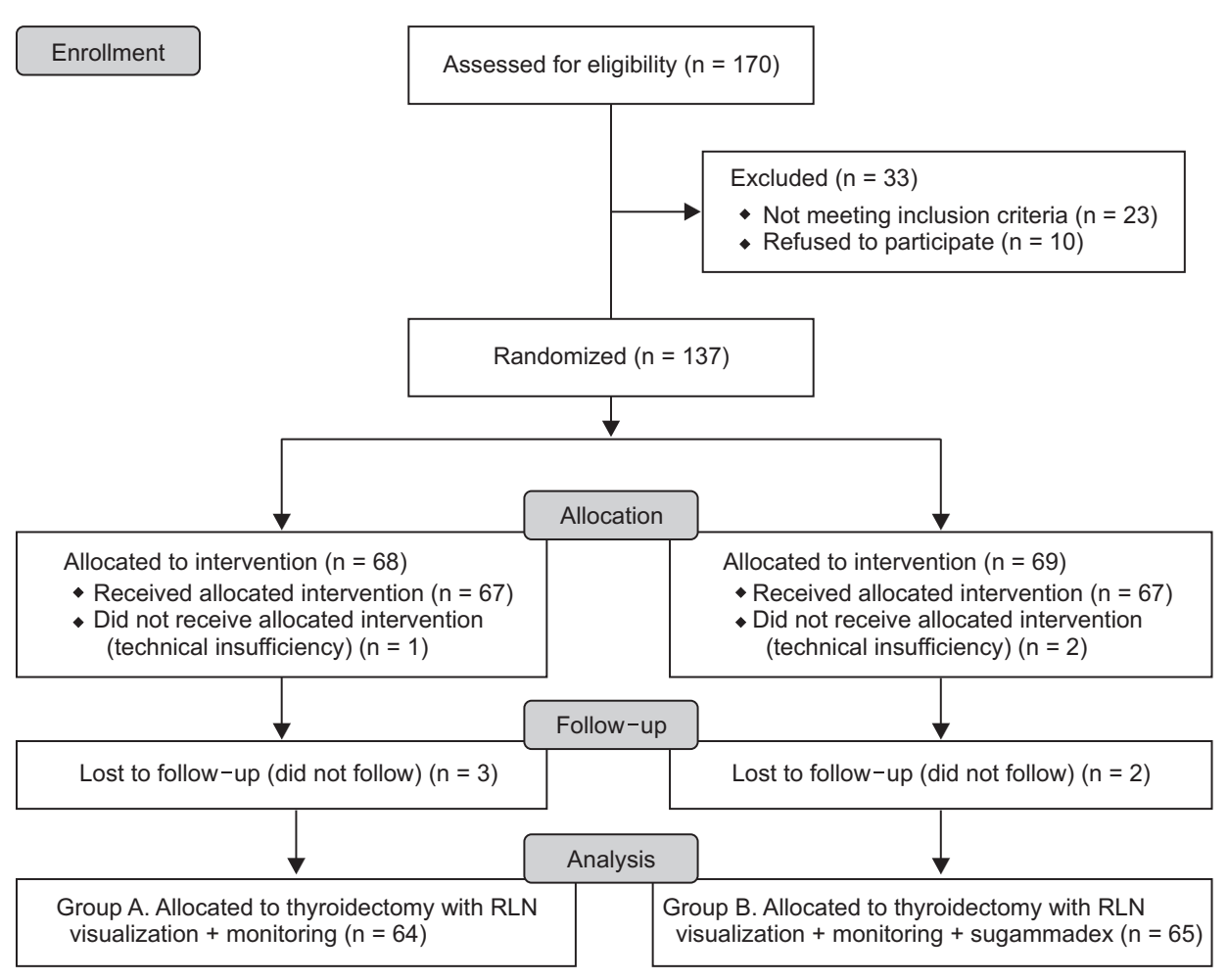

Fig. 1. Flowchart diagram of the study. RLN, recurrent laryngeal nerve. included in the computer database. The person responsible for randomization was not involved in the surgery or anesthesia team. The surgical team comprised the same members treating the patients in the 2 study groups, and patient data were collected by an independent observer.

\section{Anesthetic management}

Pre-anesthetic medication was standardized for all patients. Patients were given premedication with intravenous midazolam, up to $2 \mathrm{mg}$ if needed, upon arriving to the operating room. The patient was monitored for arterial blood pressure, oxygen saturation and electrocardiography. After oxygen was given for 3 minutes with an oxygen mask, general anesthesia was given - that is, a remifentanil $(1 \mathrm{ng} / \mathrm{mL})$, propofol $(2-3 \mathrm{mg} / \mathrm{kg})$ and fentanyl $(0.5-1 \mu \mathrm{g} / \mathrm{kg})$ infusion. Intravenous bolus dose was given consisting of $0.6 \mathrm{mg} / \mathrm{kg}$ rocuronium bromide to allow deep muscle relaxation in all patients. Subsequently, anesthesia was maintained with the infusion of sevoflurane (with a minimum alveolar concentration $[\mathrm{MAC}]$ of 0.8$)$, remifentanil $(0.05-0.2 \mu \mathrm{g} / \mathrm{kg} / \mathrm{dk})$ and an air- $\mathrm{O}_{2}$ combination of $4 \mathrm{lt} / \mathrm{min}$. No additional dose of NMBA was injected into any patient in the 2 groups. The patients were ventilated for 2 minutes using a face mask after loss of consciousness. After injection of sugammadex in group B, maintenance of anesthesia was achieved with sevoflurane (MAC between 0.9 and 1.1) and remifentanyl $(0.1-0.4 \mu \mathrm{g} / \mathrm{kg} / \mathrm{dk})$ according to each patient's heart rate and blood pressure. The $7.5-$ to $8.0-\mathrm{mm}$ diameter endotracheal tube integrated with the surface electrodes was placed by the anesthetist between the VC during intubation, and the NIM nerve monitoring system (Medtronic, Jacksonville, FL, USA) was activated. IONM equipment installation, anesthesia induction, intubation tube verification tests, and EMG measurements were all performed according to the standards described by the International Neuromonitorization Working Group (INMSG) Guidelines [19]. IONM equipment with audio and graphics monitoring documentation were used. The technique standardized by the INMSG Group was used [19]. The IONM technique included: (1) vagal nerve (VN) stimulation before lobectomy (V1); (2) RLN stimulation prior to lobectomy (R1); (3) RLN stimulation after lobectomy (R2); and (4) VN stimulation after lobectomy (V2). The intermittent stimulation technique for IONM was performed using a monopolar electrode with a pulse duration of $1 \mathrm{~mA}$ to $2 \mathrm{~mA}$, a pulse duration of $100 \mathrm{msec}$ and a nerve impulse at a frequency of $4 \mathrm{~Hz}$. If branched RLN nerves were detected, each branch was stimulated separately. Loss of signal (LOS) was defined as an EMG signal below 100 $\mu \mathrm{V}$ following stimulation of the $\mathrm{VN}$, and there was no laryngeal motion and no visible laryngeal twitching following stimulation of the ipsilateral VN [16]. To differentiate between true and false LOS, the INMSG-proposed problem-solving algorithm was employed intraoperatively [16]. A neuromapping technique was used to determine nerve damage and localization of the injury site in patients who exhibited intraoperative LOS.

Following intubation, the PNS monitor was placed on the left hand. For PNS, the TOF-Watch SX (Schering-Plow, Dublin, Ireland) was used. The electrodes of the TOF guard 
were placed close to the wrist and the ulnar nerve, and the acceleromyographic was placed on the thumb of the left hand. Electrical stimulation was performed from the TOF device; the ulnar nerve was stimulated, and the response of the adductor pollicis muscle was recorded. This ulnar stimulation was performed at a frequency of $50 \mathrm{~Hz}$ for 5 seconds. The stimulation used during the operation consisted of $0.2-\mathrm{msec}$ square-wave pulses, given as $2-\mathrm{Hz}$ TOF pulses at 15 -sec intervals. When the ratio of TOF was $>0.9$, a positive value was accepted, and TOF stimulation was terminated.

\section{Surgical technique}

All patients underwent thyroidectomy with the same surgical technique. A 4- to 5-cm Kocher incision was made, and the platysma with subplatysmal flaps was raised using electrocautery. The strap muscles were retracted for lateral exposure of the middle thyroid vein, if present, to be divided. The pyramidal lobe and the isthmus were dissected using electrocautery. During total thyroidectomy, the VN was routinely exposed on the right side first. The dissection was performed between the carotid artery and jugular vein to expose and identify the VN. IONM (V0). Values of the TOF device measurements were used to measure a signal from the VN. Surgical resection was initiated when the first signal from the right V1 $(>100 \mu \mathrm{V})$ was detected. After RLN was detected in the tracheoesopharyngeal groove, it was completely dissected from the bery ligament. The first signal (R1) was measured when RLN was first detected, and the last signal (R2) was measured after completion of the lobectomy. The RLN was always tested with a stimulation level of 1-2 mA. When 2 structures ran close together (e.g., anterior and posterior branches of the RLN, or a small artery and RLN), then the stimulation level was decreased to $0.5 \mathrm{~mA}$ because a false EMG signal might be induced by a shunt stimulus. The stimulation frequency for IONM was set to $1 \mathrm{~Hz}$, thus continuously evaluating the RLN and VN. When the initial baseline responses were higher than $100 \mu \mathrm{V}$, surgical resection was started, and a lobectomy was performed, monitoring the RLN by using IONM and protecting the parathyroids. R2 and V2 values were recorded following surgical excision. The same procedure was conducted on the other side, and the operation was completed. The left $\mathrm{V} 2$ was recorded as the final signal.

After the V1 value was taken in group A, the surgical resection process was started. A TOF response at 30, 40, 50, 60, 70,80 , and 90 minutes and after V2 values were measured and recorded.

After V0 for group B, intravenous sugammadex sodium (2 $\mathrm{mg} / \mathrm{kg}$ ) was administered, and IONM and TOF values were measured and recorded at 2 and 4 minutes. The V1 signal was accepted as positive when a wave with an amplitude higher than $100 \mu \mathrm{V}$ was obtained. If there was no response to the first standardized IONM stimulation or if the signal was less than $100 \mu \mathrm{V}$, the TOF value $>0.9$ was considered to be the technical failure of IONM. When the V1 signal was considered positive (spontaneously or after sugammadex), the dissection was started. Then, RLN was first found at the tracheoesophageal groove and then dissected completely from Berry's ligament. The first signal $(\mathrm{R} 1>100 \mu \mathrm{V})$ was obtained after the detection of RLN from RLN. When the initial baseline responses were higher than $100 \mu \mathrm{V}$, surgical resection was started, and a lobectomy was performed, monitoring the RLN by using IONM and protecting the parathyroids. R2 and V2 values were recorded following surgical excision. The same procedure was conducted on the other side, and the operation was completed. The left V2 was recorded as the final signal.

\section{Statistics}

Statistical software (JMP version 10.0.0, SAS, Cary, NC, USA) was used. Continuous variables are given as mean \pm standard deviation values or median and interquartile ranges. Categorical variables are given as frequencies and percentages. A comparison of parametric continuous variables was conducted using the Student t-test. The Mann-Whitney U-test was used for comparison of nonparametric variables. In both groups, predissection and postdissection evoked potential amplitudes on EMG values, which were evaluated using the Wilcoxon test. The chi-square test was used when comparing the categorical variables. P-values of 0.05 or below were accepted as statistically significant.

\section{RESULTS}

Among the 203 patients included in the study, total thyroidectomy was planned in 33 patients with hemitroidectomy, 166 patients with total thyroidectomy and 4 patients with lateral neck dissection. Twenty-five patients were excluded from the study because there was no possibility to use sugammadex and IONM during the operation, and 10 patients refused to participate. Eight of the patients left after 4 months of follow-up. The patient flowchart of this study is shown in Fig. 1.

Unilateral VC paralysis was confirmed in laryngoscopy performed by the postoperative day 1 by an independent otolaryngolist in 7 intraoperative cases. When the intraoperative RLN nerve map was removed in these cases, segmental type damage (type I) was detected in 4 cases and global type (type II) in 3 cases. None of the patients had any interruption in RLN structural continuity. Early RLN damage was temporary in all 7 cases $(100 \%)$.

After the VN dissection during the operation, there was no EMG signal from 2 patients in group A and 3 in group B. These patients were treated with the TOF device, which was not 
affected by a muscle relaxant. These 5 patients were excluded from the study. There were no complications associated with the surgical procedures after complete reversal of the neuromuscular blockade.

There were no significant differences in terms of age, gender, body mass index (BMI), ASA physical status classification or comorbidities in both groups (Table 1). The mean age of the patients in group A was $48.73 \pm 11.17$ years and $48.98 \pm 11.62$ years in group $B(P=0.901)$. The mean BMI of the patients in group $A$ was $28.68 \pm 3.52$ years and $28.79 \pm 4.78$ years in group $\mathrm{B}(\mathrm{P}=0.887)$ (Table 1$)$.

Preoperative diagnosis showed both benign and malignant disease, Graves' disease, recurrent goiter, multinodular goiter, DTC, DTC with neck dissection and toxic goiter. There was no significance in terms of the distribution of benign vs. malignant disease in both groups $(P=0.427$ ) (Table 1 ).

Surgical time was significantly shorter in group B $(90.78 \pm$ 13.19 minutes) than in group A (111.04 \pm 25.52 minutes) $(\mathrm{P}<$ 0.0001 ). There was no significant difference in $\mathrm{V0}$ time for the 2 groups $(\mathrm{P}=0.338)$. V1 time was significantly shorter in group $\mathrm{B}(26.07 \pm 3.26$ minutes) than in group A (50.0 \pm 8.46 minutes) $(\mathrm{P}<0.0001)$ (Table 2). Sufficient EMG response was achieved in V1 $(>100)$ stimulation with toxic goiter (20 patients in group

Table 1. Characteristics of patients

\begin{tabular}{|c|c|c|c|}
\hline Parameter & Group A $(n=64)$ & Group B $(n=65)$ & P-value \\
\hline Age (yr) & $48.73 \pm 11.17$ & $48.98 \pm 11.62$ & 0.901 \\
\hline Sex, male:female & $9: 55$ & $7: 58$ & 0.570 \\
\hline $\begin{array}{l}\text { Body mass index } \\
\left(\mathrm{kg} / \mathrm{m}^{2}\right)\end{array}$ & $28.68 \pm 3.52$ & $28.79 \pm 4.78$ & 0.887 \\
\hline RLNs at risk (n) & 128 & 130 & \\
\hline ASA PS classification & & & 0.619 \\
\hline I:II:III & $11: 49: 4$ & $12: 46: 7$ & \\
\hline Comorbidity & $21(32.81)$ & $26(40)$ & 0.396 \\
\hline DM II & 5 & 11 & \\
\hline $\begin{array}{l}\text { Renal } \\
\text { transplantation }\end{array}$ & - & 1 & \\
\hline Hypertension & 7 & 9 & \\
\hline IHD & 2 & 2 & \\
\hline COPD & 7 & 3 & \\
\hline $\begin{array}{l}\text { Preoperative } \\
\text { diagnosis }\end{array}$ & & & 0.427 \\
\hline MNG & $31(48.43)$ & $36(55.38)$ & \\
\hline Recurrent MNG & $1(1.56)$ & $1(1.53)$ & \\
\hline DTC & $10(15.62)$ & $9(13.84)$ & \\
\hline $\begin{array}{l}\text { DTC with neck } \\
\text { dissection }\end{array}$ & $2(3.12)$ & $2(3.07)$ & \\
\hline Toxic Goiter & $20(31.25)$ & $17(26.15)$ & \\
\hline
\end{tabular}

Values are presented as mean \pm standard deviation or number (\%). Group A, control group; group B, sugammadex group; RLN, recurrent laryngeal nerve; ASA PS, American Society of Anesthesiologist physical status; DM, diabetes mellitus; IHD, ischemic heart disease; COPD, chronic obstructive pulmonary disease; MNG, multinodular Goiter; DTC, differentiated thyroid carcinoma.
$B$ and 20 patients in group A). However, TOF $>0.9$ was not sufficient in these patients. TOF $>0.9$ time was significantly shorter in group B $(27.46 \pm 3.49$ minutes) than in group A (92.18 \pm 14.33 minutes) $(\mathrm{P}<0.0001)$ (Table 2). The first V1 (Right V1) EMG value was significantly higher in group $B$ than in group $A(P$ $<0.0001$ ), and the final V2 EMG value after total thyroidectomy was significantly higher in group $B$ than in group $A(P<0.045)$ (Table 2).

The EMG values before and after thyroid lobe resection in group A are summarized in Table 3. Right V1 to right V2, left $\mathrm{V} 1$ to $\mathrm{V} 2$, right $\mathrm{R} 1$ to $\mathrm{R} 2$ and left $\mathrm{R} 1$ to R2 EMG values were significantly different $(\mathrm{P}<0.001, \mathrm{P}<0.001, \mathrm{P}<0.001, \mathrm{P}=$ 0.002 , respectively) (Table 3). Likewise, the EMG values before and after thyroid lobe resection in group B are summarized in Table 3. Right V1 to right V2, left V1 to V2, right R1 to R2, and left R1 to R2 electromyography, values were not significantly different $(\mathrm{P}=0.389, \mathrm{P}=0.235, \mathrm{P}=0.092, \mathrm{P}=0.314$, respectively) (Table 3).

\section{DISCUSSION}

The standardization of technological innovations is important for safe and easy implementation of education and training $[20,21]$. Such a standardized application in correlation

Table 2. Operative values

\begin{tabular}{lccr}
\hline \multicolumn{1}{c}{ Variable } & Group A $(\mathrm{n}=64)$ & Group B $(\mathrm{n}=65)$ & P-value \\
\hline $\begin{array}{l}\text { Duration of } \\
\text { operation }\end{array}$ & $111.04 \pm 25.52$ & $90.78 \pm 13.19$ & $<0.001$ \\
(min) & & & \\
V0 time (min) & $24.32 \pm 4.32$ & $23.64 \pm 3.71$ & 0.338 \\
V1 time (min) & $50.0 \pm 8.46$ & $26.07 \pm 3.26$ & $<0.001$ \\
Transient RLN & $4(6.25)$ & $3(4.61)$ & 0.681 \\
paralysis & & & \\
Permanent RLN & $0(0)$ & $0(0)$ & \\
paralysis & & & \\
TOF $>0.9$ & $92.18 \pm 14.33$ & $27.46 \pm 3.49$ & $<0.001$ \\
value access & & & \\
time $(\min )$ & & & \\
Right $\mathrm{V} 1(\mu \mathrm{V})$ & $175.59 \pm 102.40$ & $567.13 \pm 218.90$ & $<0.001$ \\
Left $\mathrm{V} 2(\mu \mathrm{V})$ & $513.51 \pm 289.90$ & $610.38 \pm 252.63$ & 0.045 \\
V0 $(\mu \mathrm{V})$ & $7.10 \pm 12.02$ & $6.01 \pm 11.92$ & 0.356 \\
\hline
\end{tabular}

Values are presented as mean \pm standard deviation or number (\%).

Group A, control group; group B, sugammadex group; RLN, recurrent laryngeal nerve; V0 time, duration of first electromyography (EMG) value from right vagus nerve just after entering the dissection plane; $\mathrm{V} 1$ time, duration of first (initial) signal right vagus nerve; TOF, train-of-four; Right V1, first signal obtained from right vagus nerve $(>100 \mu \mathrm{V}$ ) (as the initial signal obtained before starting thyroid gland dissection); Left V2, last signal left vagus nerve (as the last signal obtained after completing the thyroid gland resection); $\mathrm{V0}$, first EMG value from right vagus nerve. 
Table 3. Comparison of intraoperative neuromonitoring values

\begin{tabular}{lcccc}
\hline Parameter & Group A & P-value & Group B & P-value \\
\hline Right V1 & $175.59 \pm 102.43$ & $<0.001$ & $567.13 \pm 218.90$ & 0.389 \\
Right V2 & $266.81 \pm 148.22$ & & $579.81 \pm 239.62$ & \\
Right LR1 & $185.39 \pm 126.06$ & $<0.001$ & $459.90 \pm 179.07$ & 0.092 \\
Right LR2 & $267.81 \pm 176.23$ & & $479.69 \pm 200.74$ & \\
Left V1 & $402.15 \pm 278.06$ & $<0.001$ & $598.78 \pm 237.31$ & 0.235 \\
Left V2 & $513.51 \pm 289.90$ & & $610.38 \pm 252.63$ & \\
Left LR1 & $359.04 \pm 251.19$ & 0.002 & $488.89 \pm 209.39$ & 0.314 \\
Left LR2 & $427.26 \pm 17.06$ & & $495.58 \pm 203.83$ & \\
\hline
\end{tabular}

Values are presented as mean \pm standard deviation.

Group A, control group; group B, sugammadex group; Right V1, first signal obtained from right vagus nerve $(>100 \mu \mathrm{V})$ (as the initial signal obtained before starting thyroid gland dissection); Right V2, VN stimulation after right lobectomy; Right LR1, RLN stimulation prior to right lobectomy; Right LR2, RLN stimulation after right lobectomy, Left V1, VN stimulation prior to left lobectomy; Left V2, last signal left vagus nerve (as the last signal obtained after completing the thyroid gland resection); Left LR1, RLN stimulation prior to left lobectomy; Left LR2, RLN stimulation after left lobectomy.

with guidelines provides an increased RLN identification rate, reduction of the severity in an RLN injury (if it should occur), detection of RLN branching or non-RLN and the intraoperative identification of nerve injury or stress [10,22]. In the thyroidectomy performed with IONM, V1 signaling was standardized [10,22]. V1 signaling prevents nerve detection, visual misidentification and provides confirmation and dissection for resection. IONM can be conducted even if the RLN is not identified by using V1 for dissection and RLN identification [23-25]. It was reported that standardized IONM or continue IONM could increase the nerve recognition rate [23,24].

In order to prevent RLN paralysis, it was emphasized that the use of IONM may be the gold standard of visual identification in thyroid surgery [4,22]. In our study, we used IONM and PNS to show that an NMB effect is returned. The IONMG suggests that stimulation of the vagus nerve and V1 signal measurement are routine procedures in thyroid surgery $[1,19]$.

Pavoni et al. [16] used a high dose of sugammadex sodium $(16 \mathrm{mg} / \mathrm{kg})$ to reverse the effect of high dosage of rocuronium $(1.2 \mathrm{mg} / \mathrm{kg})$ administered in thyroidectomy procedures in their clinical study. In this study, sufficient IONM values were reached after 70 seconds of sugammadex and a sufficient TOF $>0.9$ after 120 seconds.

The sugammadex sodium dose of $16 \mathrm{mg} / \mathrm{kg}$ is recommended for emergency situations, and a dosage of $2 \mathrm{mg} / \mathrm{kg}$ can reverse the effect of rocuronium. We used $2 \mathrm{mg} / \mathrm{kg}$ of the sugammadex sodium and obtained a complete reversal in all the patients within 3 to 4 minutes following administration of the drug.

Yamamoto et al. [26] used 2 and $4-\mathrm{mg} / \mathrm{kg}$ doses of sugammadex sodium, respectively, in their 2 groups to reverse the effect of $1 \mathrm{mg} / \mathrm{kg}$ of rocuronium. Following these doses, they recorded the TOF responses; however, sufficient TOF responses were not achieved in the group that received 2 $\mathrm{mg} / \mathrm{kg}$ of sugammadex sodium; thus, a dose of $4 \mathrm{mg} / \mathrm{kg}$ was necessary. Lu et al. [14] compared the recovery of the NMB effect with $0.6-\mathrm{mg} / \mathrm{kg}$ rocuronium by using 2 and $4 \mathrm{mg} / \mathrm{kg}$ of sugammadex sodium in pigs in 2 separate groups, respectively. They reported that $2 \mathrm{mg} / \mathrm{kg}$ of sugammadex sodium was sufficient to reverse the NMB in a rapid and safe manner. In their study, only sugammadex dosage amounts were compared. In addition, no sugammadex control group was used, and information regarding RLN paralysis is available. According to the retrospective study of Empis de Vendin et al. [27], the use of sugammadex in a good neuromuscular blocker agent was unnecessary and increased cost. However, no TOF values were validated in this study. In these 2 studies, sugammadex was used at a dosage of $2 \mathrm{mg} / \mathrm{kg}$, as in our study, and the improvement of the NMB effect was demonstrated by nerve monitoring and the use of a PNS. In a clinical study involving 120 patients by Empis de Vendin et al., sugammadex injections were performed in 15 patients whose V1 value could not be obtained, and no control group was formed, as in the study of Lu et al. In their study, the number of patients in their 2 groups was disproportionate and therefore may not provide accurate results. In their study, the mean duration of V1 (100 mV) uptake was 42 minutes in the nonsugammadex group and 40 minutes in the sugammadex group. However, in our study, V0 was 23.8 minutes and V1 was 26 minutes in the sugammadex group, while V0 was 24 minutes and V1 was 50 minutes in the control group, and the difference between the groups was significant. As the elimination of NMB drugs differs from person to person, a positive response would be obtained during surgery, but in the study of Empis de Vendin et al. There is no a strict sugammadex administration protocol. In our methods section, the sugammadex administration has been standardized. Furthermore, objectivity is obtained because of the prospective and randomized study design. We also included a control group in the study, and all results were compared between study and control groups, which contrasts previous studies with human subjects.

Since the total operation times were not compared in these 22 studies, the benefit of sugammadex could not be fully established. The major limitation of these 2 studies is the retrospective design. To the best of our knowledge, our study is the first randomized prospective clinical trial on human subjects on this topic.

In our clinical study, the VN was detected, the first EMG signal (VO) was taken and 2-mg/kg sugammadex was applied. After giving $0.6 \mathrm{mg} / \mathrm{kg}$ of rocuronium for general anesthesia induction, $23.64 \pm 3.71$ minutes had elapsed. Amplitudes 
during IONM usually showed variability from patient to patient [22]. Factors other than the NMB that may have affected the evoked EMG amplitude were endotracheal tube position, manipulation of the gland or trachea during measurement, moisture or irrigation of the surgical field, sheated nerve within the fascia that spoils probe to nerve contact and temperature $[23,28]$. In this study, the reasons for the high standard deviation of $\mathrm{V} 1$ and $\mathrm{V} 2$ signals can be explained by these factors. The comparability of the V1 and V2 signals indicates that sugammadex permits the rapid restoration of neuromuscular function suppressed by rocuronium at the beginning of the operation. The use of sugammadex can shorten the time of recovery from NMB. In this study, sugammadex was found to be effective in dose-dependent NMB, and 2.0 to $4.0 \mathrm{mg} / \mathrm{kg}$ was effective in facilitating extubation [29].

In group $B$, there was no significant difference between V1 and V2 $(P=0.389)$, and the V1 time was significantly shorter in group $B(26.07 \pm 3.26$ minutes) than in group A (50.0 \pm 8.46 minutes) $(\mathrm{P}<0.001)$. In Group $A$, there was a significant difference between V1 and V2 $(\mathrm{P}<0.001)$, and the first V1 (Right V1) EMG value was significantly higher in group $B$ than in Group A ( $<$ 0.001) (Fig. 2). In our clinical study, when the V1 value was obtained in group $B$, there was a sufficient TOF $>0.9$ (Fig. 3). When the V1 value was obtained in group A, there was not a sufficient TOF $>0.9$. TOF $>0.9$ time was significantly shorter in group B (27.46 \pm 3.49 minutes) than in group A (92.18 \pm 14.33) $(P<0.001)$. Also, in group $A$, there was a significant difference between the V1 time (50.0 \pm 8.46 minutes) and the TOF $>0.9$ time $(92.18 \pm 14.33)$ (Fig. 4). However, there was no significant difference between the V1 time and the TOF $>0.9$ time (27.46 \pm 3.49 minutes) in group B (Table 2). Therefore, there was a significant difference between $\mathrm{V} 1$ and TOF $>0.9$ in both groups.

Many studies have compared RLN paralysis incidences with IONM and direct nerve visualization. Pisanu et al. [30]

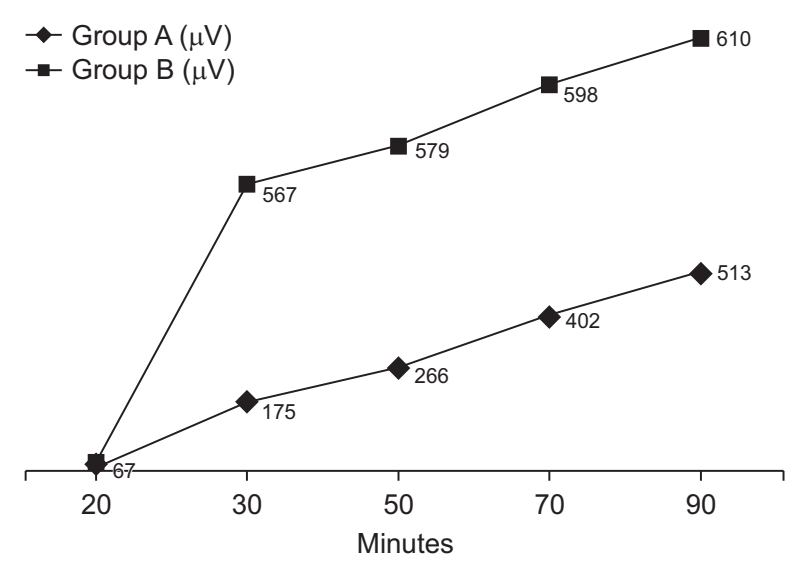

Fig. 2. The electromyography (EMG) values of the vagus nerve. Group A, control group; group B, sugammadex group. reported a meta-analysis comparing 20 studies in terms of IONM use and direct visualization. A total of 16,517 patients were included in this meta-analysis, and the incidence of RLN paralysis was $3.47 \%$ in their IONM group and $3.67 \%$ in their direct vision group with no significant difference. Contention remains regarding whether it is excessive trust in the tool or false signals from vascular structures and bleeding that cause the inability to reduce RLN paralysis incidence with IONM use $[3,10]$. Although the values for vagus over $100 \mu \mathrm{V}$ were accepted as positive in some patients, we found that the TOF response for the V1 values were higher than this point but lower than 0.9 .

In a large-scale meta-analysis study of Pisanu et al. [30], 20 studies were performed, and 7 studies provided data about the duration times of the surgeries. In the IONM group, shorter surgery times were determined in 2 studies, while the surgery

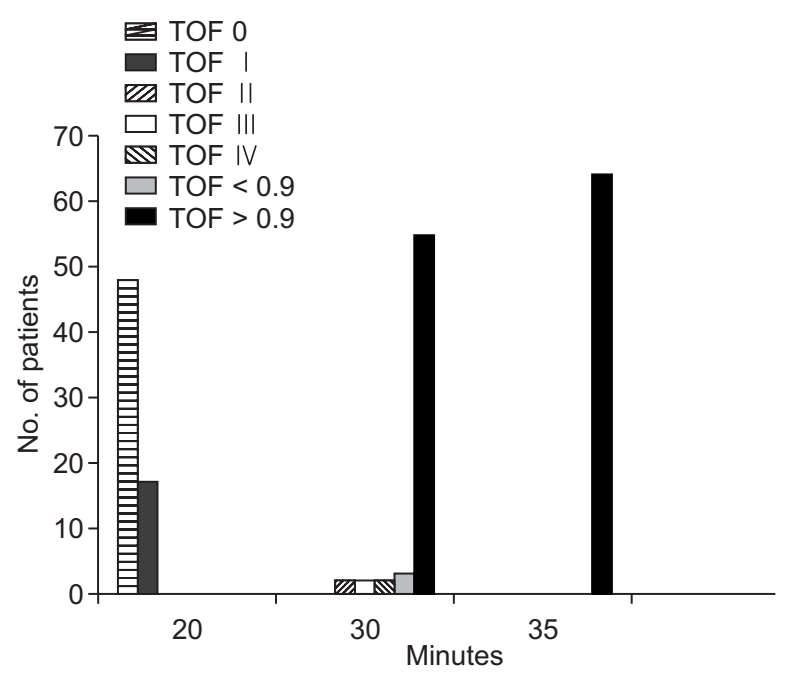

Fig. 3. Train-of-four (TOF) values of group B. Group B, sugammadex group.

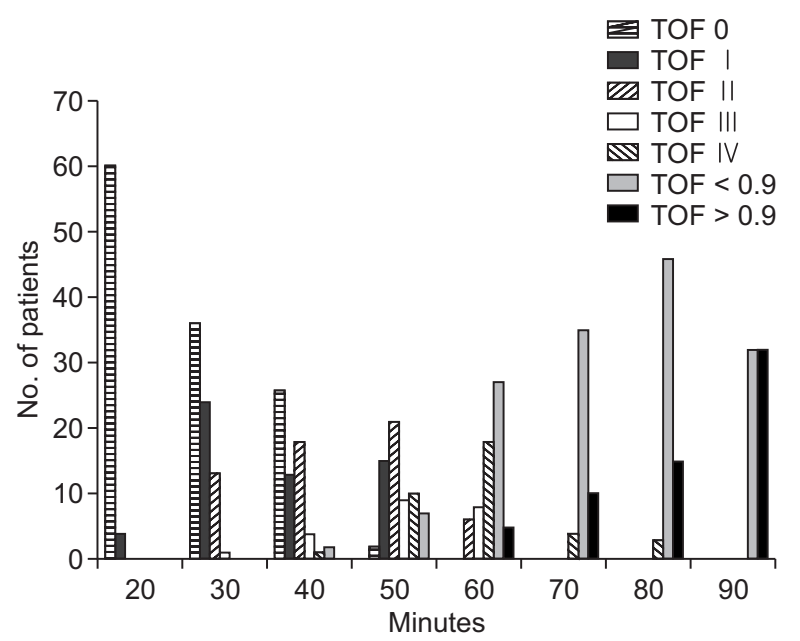

Fig. 4. Train-of-four (TOF) values of group A. Group A, control group. 
time was shorter in 5 studies involving the direct visualization method. In 2 studies, the principle of reversing rocuroniuminduced NMB with sugammadex was conducted, but no information was given about the surgery duration [14,27]. In our study, the duration times for surgeries were significantly shorter in group $B$ than in group $A(P<0.001)$.

In conclusion, neuromonitoring may be a useful aid to ensure the functional and structural integrity of the nerve in cases of anatomic dissection difficulty, especially with large tissue masses, abnormal nerve course and previous surgery. In addition, IONM is a secure and efficient method for the diagnosis of an RLN in thyroid surgery. IONM can offer safe operation with high accuracy, specificity and sensitivity.
Sugammadex provides efficient and rapid recovery of nondepolarizing neuromuscular block generated by rocuronium used to facilitate intubation. The use of sugammadex enables shorter operative times due to the rapid recovery of the neuromuscular blockade. Furthermore, this reversal of neuromuscular block allows intraoperative neuromonitorization to be used effectively and safely during thyroid surgery.

\section{CONFLICTS OF INTEREST}

No potential conflict of interest relevant to this article was reported.

\section{REFERENCES}

1. Steurer M, Passler C, Denk DM, Schneider B, Niederle B, Bigenzahn W. Advantages of recurrent laryngeal nerve identification in thyroidectomy and parathyroidectomy and the importance of preoperative and postoperative laryngoscopic examination in more than 1000 nerves at risk. Laryngoscope 2002;112:124-33.

2. Dionigi G, Dionigi R. Standardization of intraoperative neuromonitoring of recurrent laryngeal nerve in thyroid operation: to the editor. World J Surg 2010;34:2794-5.

3. Dralle H, Sekulla C, Lorenz K, Brauckhoff M, Machens A; German IONM Study Group. Intraoperative monitoring of the recurrent laryngeal nerve in thyroid surgery. World J Surg 2008:32:1358-66.

4. Kim SJ, Lee KE, Oh BM, Oh EM, Bae DS, Choi JY, et al. Intraoperative neuromonitoring of the external branch of the superior laryngeal nerve during robotic thyroid surgery: a preliminary prospective study. Ann Surg Treat Res 2015;89:233-9.

5. Barczynski M, Konturek A, Cichon S. Randomized clinical trial of visualization versus neuromonitoring of recurrent laryngeal nerves during thyroidectomy. $\mathrm{Br} \mathrm{J}$ Surg 2009:96:240-6.

6. Zheng S, Xu Z, Wei Y, Zeng M, He J. Effect of intraoperative neuromonitoring on recurrent laryngeal nerve palsy rates after thyroid surgery--a meta-analysis. J Formos
Med Assoc 2013;112:463-72.

7. Angelos P. Recurrent laryngeal nerve monitoring: state of the art, ethical and legal issues. Surg Clin North Am 2009;89: 1157-69.

8. Randolph GW, Kamani D. Intraoperative electrophysiologic monitoring of the recurrent laryngeal nerve during thyroid and parathyroid surgery: experience with 1,381 nerves at risk. Laryngoscope 2017; 127:280-6.

9. Shindo M, Chheda NN. Incidence of vocal cord paralysis with and without recurrent laryngeal nerve monitoring during thyroidectomy. Arch Otolaryngol Head Neck Surg 2007;133:481-5.

10. Chiang FY, Lee KW, Chen HC, Chen HY, Lu IC, Kuo WR, et al. Standardization of intraoperative neuromonitoring of recurrent laryngeal nerve in thyroid operation. World J Surg 2010;34:223-9.

11. Lee C, Jahr JS, Candiotti KA, Warriner B, Zornow MH, Naguib M. Reversal of profound neuromuscular block by sugammadex administered three minutes after rocuronium: a comparison with spontaneous recovery from succinylcholine. Anesthesiology 2009;110:1020-5.

12. Marusch F, Hussock J, Haring G, Hachenberg T, Gastinger I. Influence of muscle relaxation on neuromonitoring of the recurrent laryngeal nerve during thyroid surgery. Br J Anaesth 2005;94:596600.

13. Wu CW, Wang MH, Chen CC, Chen HC, Chen HY, Yu JY, et al. Loss of signal in recurrent nerve neuromonitoring: causes and management. Gland Surg 2015:4:1926.

14. Lu IC, Wu CW, Chang PY, Chen HY, Tseng KY, Randolph GW, et al. Reversal of rocuronium-induced neuromuscular blockade by sugammadex allows for optimization of neural monitoring of the recurrent laryngeal nerve. Laryngoscope 2016;126:1014-9.

15. Kopman AF, Zank LM, Ng J, Neuman GG. Antagonism of cisatracurium and rocuronium block at a tactile train-of-four count of 2: should quantitative assessment of neuromuscular function be mandatory? Anesth Analg 2004:98:102-6.

16. Pavoni V, Gianesello L, Martinelli C, Horton A, Nella A, Gori G, et al. Recovery of laryngeal nerve function with sugammadex after rocuronium-induced profound neuromuscular block. J Clin Anesth 2016;33:14-9.

17. Kovac AL. Sugammadex: the first selective binding reversal agent for neuromuscular block. J Clin Anesth 2009;21:444-53.

18. Duvaldestin P, Kuizenga K, Saldien V, Claudius C, Servin F, Klein J, et al. A randomized, dose-response study of 
sugammadex given for the reversal of deep rocuronium- or vecuronium-induced neuromuscular blockade under sevoflurane anesthesia. Anesth Analg 2010;110: 74-82.

19. Barczynski M, Randolph GW, Cernea CR, Dralle H, Dionigi G, Alesina PF, et al. External branch of the superior laryngeal nerve monitoring during thyroid and parathyroid surgery: International Neural Monitoring Study Group standards guideline statement. Laryngoscope 2013;123 Suppl 4:S1-14.

20. Dralle H, Lorenz K. Intraoperative neuromonitoring of thyroid gland operations: Surgical standards and aspects of expert assessment. Chirurg 2010;81:612-9.

21. Gonzalez-Sanchez C, Franch-Arcas G, Gomez-Alonso A. Morbidity following thyroid surgery: does surgeon volume matter? Langenbecks Arch Surg 2013:398: 419-22.

22. Randolph GW, Dralle H; International Intraoperative Monitoring Study Group, Abdullah H, Barczynski M, Bellantone R, et al. Electrophysiologic recurrent laryngeal nerve monitoring during thyroid and parathyroid surgery: international standards guideline statement. Laryngoscope 2011;121 Suppl 1:S1-16.

23. Dionigi G, Kim HY, Wu CW, Lavazza M, Ferrari C, Leotta A, et al. Vagus nerve stimulation for standardized monitoring: technical notes for conventional and endoscopic thyroidectomy. Surg Technol Int 2013:23:95-103.

24. Wu CW, Dionigi G, Chen HC, Chen HY, Lee KW, Lu IC, et al. Vagal nerve stimulation without dissecting the carotid sheath during intraoperative neuromonitoring of the recurrent laryngeal nerve in thyroid surgery. Head Neck 2013:35:1443-7.

25. Chiang FY, Lu IC, Tsai CJ, Hsiao PJ, Lee $\mathrm{KW}, \mathrm{Wu} \mathrm{CW}$. Detecting and identifying nonrecurrent laryngeal nerve with the application of intraoperative neuromonitoring during thyroid and parathyroid operation. Am J Otolaryngol 2012:33:1-5.

26. Yamamoto $S$, Yamamoto Y, Kitajima O, Maeda T, Suzuki T. Reversal of neuromuscular block with sugammadex: a comparison of the corrugator supercilii and adductor pollicis muscles in a randomized dose-response study. Acta Anaesthesiol
Scand 2015:59:892-901.

27. Empis de Vendin O, Schmartz D, Brunaud L, Fuchs-Buder T. Recurrent laryngeal nerve monitoring and rocuronium: a selective sugammadex reversal protocol. World J Surg 2017;41:2298-303.

28. Lu IC, Chu KS, Tsai CJ, Wu CW, Kuo WR, Chen HY, et al. Optimal depth of NIM EMG endotracheal tube for intraoperative neuromonitoring of the recurrent laryngeal nerve during thyroidectomy. World J Surg 2008:32:1935-9.

29. Suy K, Morias K, Cammu G, Hans P, van Duijnhoven WG, Heeringa M, et al. Effective reversal of moderate rocuronium- or vecuronium-induced neuromuscular block with sugammadex, a selective relaxant binding agent. Anesthesiology 2007;106:283-8.

30. Pisanu A, Porceddu G, Podda M, Cois A, Uccheddu A. Systematic review with meta-analysis of studies comparing intraoperative neuromonitoring of recurrent laryngeal nerves versus visualization alone during thyroidectomy. J Surg Res 2014;188:152-61. 